\title{
NOVEL INTEGRATING SOLID STATE DETECTOR WITH SEGMENTATION FOR SCANNING TRANSMISSION SOFT X-RAY MICROSCOPY*
}

\author{
M. Feser ${ }^{\mathrm{a}}$, C. Jacobsen ${ }^{\mathrm{a}}$, P. Rehak ${ }^{\mathrm{b}}$, \\ G. De Geronimo ${ }^{b}$, P. Holl ${ }^{c}$, and L. Strüder ${ }^{d}$ \\ ${ }^{2}$ Dept. Physics \& Astronomy, SUNY, Stony Brook, NY \\ ${ }^{b}$ Brookhaven National Laboratory, Upton, NY \\ ${ }^{\circ} \mathrm{KETEK} \mathrm{GmbH}$ \\ ${ }^{\mathrm{d}}$ Max-Planck-Institut für extraterrestrische Physik
}

July, 2001

*Work supported in part by the U.S. Department of Energy Contract No. DE-AC02-98CH10886. 


\title{
Novel integrating solid state detector with segmentation for scanning transmission soft $x$-ray microscopy
}

\author{
Michael Feser $^{* *_{a}}$, Chris Jacobsen ${ }^{* a}$, Pavel Rehak ${ }^{* * b}$, Gianluigi DeGeronimo ${ }^{b}$, Peter Holl ${ }^{* * * *}$, \\ Lothar Strïder ${ }^{* * * * d}$ \\ ${ }^{a}$ Department Of Physics And Astronomy, State University Of New York At Stony Brook; \\ ${ }^{b}$ Instrumentation Division, Brookhaven National Laboratory; \\ ${ }^{\mathrm{c}} \mathrm{KETEK} \mathrm{GmbH} ;{ }^{\mathrm{d}}$ Max-Planck-Institut für extraterrestrische Physik
}

\begin{abstract}
An integrating solid state detector with segmentation has been developed that addresses the needs in scanning transmission $\mathrm{x}$-ray microscopy below $1 \mathrm{keV}$ photon energy. The detector is not cooled and can be operated without an entrance window which leads to a total photon detection efficiency close to $100 \%$. The chosen segmentation with 8 independent segments is matched to the geometry of the STXM to maximize image mode flexibility. In the bright field configuration for $1 \mathrm{~ms}$ integration time and $520 \mathrm{eV}$ $\mathrm{x}$-rays the rms noise is 8 photons per integration.
\end{abstract}

Keywords: X-ray microscopy, soft x-ray detectors

\section{INTRODUCTION}

Scanning transmission X-ray microscopes (STXMs) use Fresnel zone plates (FZPs) to focus monochromatic $\mathrm{x}$-rays to a diffraction limited spot. A sample is raster scanned through the focus and a detector measures the transmitted $\mathrm{x}$-ray flux. For a review see ${ }^{1}$ and for latest developments ${ }^{2}$.

Much effort has been put into the fabrication of Fesnel zone plates to achieve the smallest focus possible, because the size of the focus ultimately limits the spatial resolution of the STXM. Current FZPs ${ }^{3,4,10}$ achieve focal spots smaller than $40 \mathrm{~nm}$ in diameter with efficiencies close to the theoretical limit. It would be desirable to push this further towards the wavelength limit of $x$-rays, which for soft $x$-rays in the water window is roughly $2-4 \mathrm{~nm}$, but challenges in nanofabrication, in particular the necessary high aspect ratio of the zones, slow down the progress.

Not too much attention has been paid to the development of detectors in the past, even though commercial detectors do not match well the requirements of a STXM. In particular for x-ray energies in the soft x-ray regime below $1 \mathrm{keV}$ detection becomes even more problematic due to the high absorption of soft $\mathrm{x}$-rays in solid materials and the small number of primary electrons produced in detection systems in which x-rays are converted to charge. In contrast to commercially available detectors which are slow, a STXM requires a fast detector capable of measuring a large number of photons within a short time and does not require energy resolution, since monochromatic $x$-rays are a prerequisite of using diffractive optics (FZPs).

In this paper we first define the detector requirements of a STXM, which includes segmentation of the sensitive area in a small number of segments, which we will examine in some more detail. We will show that integrating detectors can perform as well as counting detectors and that the loss in performance using a system that is not cooled is acceptable and allows operation without an entrance window. The charge

\footnotetext{
* Michael.Feser@sunysb.edu; phone 1631 344-4723; fax 1631 632-8101;

http://www.physics.sunysb.edu; Department Of Physics And Astronomy, SUNY Stony Brook, Stony Brook, NY, USA 11794-3800;

***hak2@bnl.gov; phone 1631 344-3964; fax 1631 344-5773; http://www.bnl.gov; Instrumentation Division, Brookhaven National Laboratory, Building 535B, Upton, NY, USA 11973

**** peter.holl@ketek.net; phone 4989 8394000, fax 4989 83940013; http://www.ketek.net; KETEK GmbH, Am Isarbach 30, 85764 Oberschleissheim, Germany

****** Its@hll.mpg.de; phone 4989 8394000; fax 4989 83940013; http://www.hll.mpg.de; Max-PlanckInstitut für extraterrestrische Physik, Giessenbachstrasse, 85740 Garching, Germany
} 
integrating silicon detector is then described and we conclude with the measured performance and ideas about the use of this detector at higher $\mathrm{x}$-ray energies up to $12 \mathrm{keV}$.

\section{DETECTOR REQUIREMENTS FOR A STXM}

In a STXM X-rays are focused by a FZP to a diffraction limited spot through which the sample is raster scanned. It is necessary to use an order sorting aperture behind the FZP in connection with a large central gold stop on the FZP to only allow radiation that is focused into the $1^{\text {st }}$ diffraction order to reach the sample (Figure 6). The intensity pattern on a detector somewhere behind the sample is then an annulus apart from scattering and phase effects introduced by the specimen, which we will discuss below. The job of the detector is to measure how many photons reach the detector during the pixel dwell time which is the time the beam dwells on one sample position. The length of the pixel dwell time is determined by the available $\mathrm{x}$-ray flux and the number of photons that are needed per pixel to measure a sample property with a desired signal to noise ratio. It is desirable to keep the pixel dwell time as short as possible to reduce the collection time for one image which is given by the product of the number of pixels recorded and the pixel dwell time. Thus it is important for the detector to be capable of handling the highest $\mathrm{x}$-ray flux available with high detection efficiency. High detection efficiency also helps to keep the radiation dose to the specimen as low as possible.

A linear response of the detector is crucial for quantitative experiments. Counting detectors suffer from nonlinearity effects at high count rates, which need to be corrected for and which can be irreproducible.

Since the STXM ideally is a user instrument and is not operated by detector specialists, the detection system should be reliable, stable, and easy to use and not require a lot of maintenance.

Demonstration experiments in the recent past ${ }^{5,6,7,14}$ focused on the fact that the illumination pattern in the detector plane can contain additional information like local phase gradients of the sample. To implement these modes of operation as standard methods a segmented detector is needed that can perform the standard imaging mode as well as additional ones without the need of special reconfiguration of the STXM.

\section{IMAGING MODES AND DETECTOR SEGMENTATION}

The standard operating mode in a STXM is incoherent bright field imaging for which all $\mathrm{x}$-rays transmitted through the sample are detected by a large area detector (Figure 4a). Large area detectors that have been successfully used with a STXM include phosphor screens coupled to photomultiplier tubes, gas proportional counters and avalanche photodiodes. We will refer to these below as large area detectors.

If the numerical aperture of the zone plate and the numerical aperture of the detector (Figure 4b) is matched, the $\mathrm{x}$-ray detection is limited to the pupil of the illumination in the detector plane. In analogy to visible light microscopy this improves the imaging characteristics of the microscope ${ }^{11,12}$. This can be achieved by having an annular detector segment, which is accurately positioned behind the specimen. For large area detectors this could be achieved by aperturing the detector with a pinhole, but proper positioning behind the sample has to be achieved, which has not been readily available up to now.

For Nomarski differential interference contrast (DIC) two wave fronts that are slightly tilted with respect to each other are produced by using either a beamsplitter in front of the $\mathrm{FZP}^{6}$ or a zone plate doublet. The interference pattern of these two wave fronts in the detector plane, which is superimposed on the annulus, is chosen to be centered on a bright fringe and shifts of the fringe (in this case up and down) are proportional to the local phase gradient of the sample. The fringe shift can be detected by splitting the inner sensitive area e.g. into three segments as shown in Figure 4c.

DIC can also be implemented by using a quadrant detector that measures the shift of the "center of mass" of the intensity on the detector ${ }^{14}$. Since the phase gradient information will be mostly located close to the edge of the illumination pupil an annular shaped detector ring with 4 quadrant detectors can be used advantageously (Figure 4d).

Especially for biological applications labeling techniques play an important role to visualize function in biological samples. Fluorescence labeling is a standard technique in visible light microscopy. To use labèling techniques in a STXM, an antibody that binds specifically to a biological structure can be conjugated with a strong $x$-ray scatterer like a gold sphere that has the diameter of the $x$-ray focus ${ }^{5}$. When the $x$-ray focus is on the sphere there will be an appreciable amount of radiation scattered out of the 
illumination pupil on the detector and the signal from the scattered $\mathrm{x}$-rays can be detected by the 4 quadrant detectors mentioned above or a large segment surrounding the quandrant segments (Figure 4e). In conclusion we see that the imaging modes described above are compatible with just one detector segmentation design (Figure 4a-e), which we adapt for the charge integrating silicon detector. A $400 \mu \mathrm{m}$ diameter inner segment that is split into three vertical sections is used for bright field and Nomarski interference contrast. Four quadrant segments with an outer diameter of $600 \mu \mathrm{m}$ are used for dark field and differential interference contrast. One large segment surrounds the others with a diameter of $1200 \mu \mathrm{m}$ that can be used for dark field detection.

\section{COMPARISON OF COUNTING DETECTORS WITH INTEGRATING DETECTORS}

The ideal detector for a STXM would be a counter (with segmentation) that detects every $\mathrm{x}$-ray photon that is incident on the detector. The relative fluctuation of the number of photons detected within the pixel dwell time at an average photon number $\bar{N}$ follows a Poissonian statistic and is called photon shot noise:

$$
\frac{\sqrt{\operatorname{var}(N)}}{\bar{N}}=\frac{1}{\sqrt{\bar{N}}}
$$

A real counter has a finite detection efficiency $\varepsilon$ and we can write:

$$
\frac{\sqrt{\operatorname{var}(N)}}{\bar{N}}=\frac{1}{\sqrt{\varepsilon \cdot \overline{N_{i n}}}}
$$

Where $\bar{N}$ is the number of photons as detected by the detector and $\bar{N}_{i n}$ is the number of photons incident on the detector. For large photon counts the efficiency is a function of $\bar{N}$ due to the dead time of the counter, or the time structure of the x-ray source and the dependence of $\varepsilon$ on $\bar{N}$ has to be considered. For the National Synchrotron Light Source at Brookhaven National Laboratory $\mathrm{x}$-ray photons arrive in $20 \mathrm{~ns}$ intervals within a $0.2 \mathrm{~ns}$ time window. Current counters cannot count photons that arrive within the same bunch.

For an integrating detector that consists of a medium in which the conversion of $\mathrm{x}$-rays to charge takes place and charge integrating readout electronics, the fluctuation in the number of detected photons $\bar{N}$ can be written as:

$$
\frac{\sqrt{\operatorname{var}(N)}}{\bar{N}}=\sqrt{\frac{1+\frac{F \cdot w}{E}}{\varepsilon \cdot \bar{N}_{i n}}+\left(\frac{E N C \cdot w}{\varepsilon \cdot \bar{N}_{i n} \cdot E}\right)^{2}}
$$

The statistics of the conversion process of x-rays to electric charge introduce a term $F \cdot w / E$, in which $F$ is the Fano factor of the conversion medium (0.1-0.2 for silicon), $w$ the energy necessary to produce an electron hole pair in the conversion medium ( $3.6 \mathrm{eV}$ for silicon) and $E$ the x-ray energy. One can see that this term effectively decreases the efficiency of the detector system, but that for soft $x$-rays this term is on the order of $10^{-3}$ and can therefore be neglected. The other additive term under the square root is introduced by the noise (Equivalent noise charge ENC) associated with the readout electronics and noise arising from the dark current. This term is important when the charge produced by the $\mathrm{x}$-rays is on the same order or smaller than the ENC.

Figure 1 shows a comparison between a hypothetical $100 \%$ efficient counter that is only limited by the time structure of the NSLS $x$-ray source, a real gas proportional counter that is in use on the NSLS STXM IV microscope $e^{9}$ and the new integrating silicon detector in bright field detection mode for $1 \mathrm{~ms}$ dwell time and $360 \mathrm{eV}$ photons.

At the very lowest photon rates, the proportional counter delivers minimum noise, but the near-100\% efficiency of the integrating detector makes up for its electronic noise relative to the $50 \%$ efficient gas counter at photon rates greater than $20 \mathrm{kHz}$ (at $1 \mathrm{~ms}$ dwell time). 


\section{THE INTEGRATING SILICON DETECTOR WITH SEGMENTATION}

The detector chip (Figure 5) is made of $300 \mu \mathrm{m}$ thick high resistivity n-type silicon. It was fabricated in a planar technology adapted for detector production ${ }^{13}$. Rectifying $p / n$ junctions with separate current readout have been patterned by a photolithographic process on one side. The reverse side has an ohmic $\mathrm{n}^{+}$ contact for the application of a positive bias voltage to control the depletion region. Local depletion that is sufficient for illumination with soft $\mathrm{x}$-rays below $1 \mathrm{keV}$ from the front ( $\mathrm{p} / \mathrm{n}$ junction) side has been achieved with a bias voltage of $+1.5 \mathrm{~V}$. Full depletion of the volume is reached with a bias of $+90 \mathrm{~V}$ on the backside needed for illumination from the back side.

The chip is mounted and bonded to a ceramic carrier (Figure 3 upper left images) that allows the interchange of silicon chips in the detector package. The chip is not cooled, but is instead operated at ambient room temperature, which permits to use the detector without an (absorbing) entrance window and close to $100 \%$ detection efficiency owing to the shallow implants.

The lower images in Figure 3 show the detector package, which has been designed to fit the geometry of the microscope that required the front to be small (square 40 by $40 \mathrm{~mm}$ ). A simplified schematic of the charge integrating electronics is shown in Figure 2. The input from the detector is at the left side. At the beginning of each integration period a FET switch resets the feedback capacitor of the preamplifier. The output voltage of the preamplifier is stored on a capacitor on the positive input of the instrumentation amplifier by closing the corresponding switch for a short time bringing the output voltage of the instrumentation amplifier to 0 . When the switch is opened the output of the instrumentation amplifier rises linearly with the charge collected on the feedback capacitance of the preamplifier. At the end of the integration cycle a sample and hold circuit stores the instrumentation amplifier output for readout by the microscope analog to digital converter and a new integration cycle is initiated. For the operation of the STXM it is important that the clocking of the detector is set by the STXM electronics and not by the detector itself, because the pixel dwell times can vary continuously over a scan line.

\section{CONCLUSION}

A prototype of the integrating silicon detector with segmentation has been installed on the STXM IV ${ }^{9}$ soft $\mathrm{x}$-ray scanning transmission microscope at the National Synchrotron Light Source. We have measured the efficiency of the detector to be $94 \%$ for $520 \mathrm{eV}$ x-rays. The rms noise of the detector in bright field mode (sum of inner three segments) is 8 photons for $520 \mathrm{eV}$ and $1 \mathrm{~ms}$ dwell time. The rms noise is indirectly proportional to energy, which favors higher energies. In principle it should be possible to use this detector for energies up to $12 \mathrm{keV}$ for which the $300 \mu \mathrm{m}$ chip thickness still detects more than $70 \%$ of the incident $\mathrm{x}$-rays. The total number of photons $(360 \mathrm{eV})$ that can be detected in one pixel dwell time is $5^{*} 10^{4}$ for the bright field configuration. For higher photon energies this number is smaller, but it can be adjusted by a hardware modification of the feedback capacitance of the preamplifier.

We are operating the detector in the backillumination mode, since we do not observe any radiation damage in this mode. For illumination from the front (the $\mathrm{p} / \mathrm{n}$ junction side) we observed radiation damage which is attributed to a surface oxide layer. A new fabrication process featuring a thin Aluminum layer on top might solve this problem in the future. The detector has 8 independent channels which can be used for differential phase contrast and dark field experiments. First experiments are underway on which we will report soon.

\section{ACKNOWLEDGMENTS}

D. Pinelli (BNL, Instrumentation Division) mounted and bonded the detector chips and assembled the detector electronics and mechanics. The printed circuit boards of the readout electronics have been laid out by Ronald Ryan (BNL, Instrumentation Division).

We would like to thank G. Smith (BNL, Instrumentation Division) for fruitful discussions and for bringing the people of this collaboration together. 


\section{REFERENCES}

1. J. Kirz, C. Jacobsen, and M. Howells, Soft $x$-ray microscopes and their biological applications, Quarterly Reviews of Biophysics, 28(1), pp. 33-130, 1995.

Also available as Lawrence Berkeley Laboratory report LBL-36371

2. W. Meyer-Ilse, T. Warwick, and D. Attwood, eds., X-ray Microscopy: Proceedings of the Sixth International Conference, (Melville, NY), American Institute of Physics, 2000

3. S. Spector, C. Jacobsen, and D. Tennant, Process optimization for production of sub-20 sm soft x-ray zone plates, Journal of Vacuum Science and Technology, 1997, B 15(6), pp. 2872-2876

4. D. Tennant, et al., Electron beam lithography of Fresnel zone plates using a rectilinear machine and trilayer resists, in ${ }^{2}$, pp. 601-606

5. H. N. Chapman, C. Jacobsen, and S. Williams, A characterisation of dark-field imaging of colloidal gold labels in a scanning transmission $x$-ray microscope, Ultramicroscopy, 62(3), pp. 191-213, 1996

6. F. Polack, D. Joyeux, et al., Demonstration of phase contrast in scanning transmission $x$-ray microscopy: comparison of images obtained at NSLS X-1A with numerical simulations, in ${ }^{2}$, pp. 573580

7. W. J. Eaton, et al., Configured detector system for STXM imaging, in ${ }^{2}$, pp. 452-457

8. T. Wilhein, et al., Differential interference contrast $x$-ray microscopy with submicron resolution, Applied Physics Letters 78(14), pp. 2082-2084, 2001

9. M. Feser, T. Beetz, M. Carlucci-Dayton, and C. Jacobsen, Instrumentation advances and detector development with the Stony Brook scanning transmission $x$-ray microscope, in ${ }^{2}, \mathrm{pp} .367-372$

10. M. Peuker, High-efficiency nickel phase zone plates with $20 \mathrm{~nm}$ minimum outermost zone width, Applied Physics Letters 78(15), pp. 2208-2210, 2001

11. M. Born, and E. Wolf, Principles of optics, pp. 522-524, Cambridge University Press, UK, Sixth Ed., 1980

12. S. Vogt, et al., Dark field $x$-ray microscopy: the effects of condenser/detector aperture, Ultramicroscopy 87, pp. 25-44, 2001

13. J. Kemmer, Fabrication of low noise silicon radiation detectors by the planar process, Nuclear Instruments and Methods A 169, pp. 499-502, 1980

14. J. Palmer, G. Morrison, Differential phase contrast imaging in $x$-ray microscopy, in ${ }^{15}$, pp. $278-280$

15. A. Michette, G. Morrison, and C. Buckley, X-ray microscopy III, Springer series in optical sciences 67, Springer Verlag, Berlin, 1992 


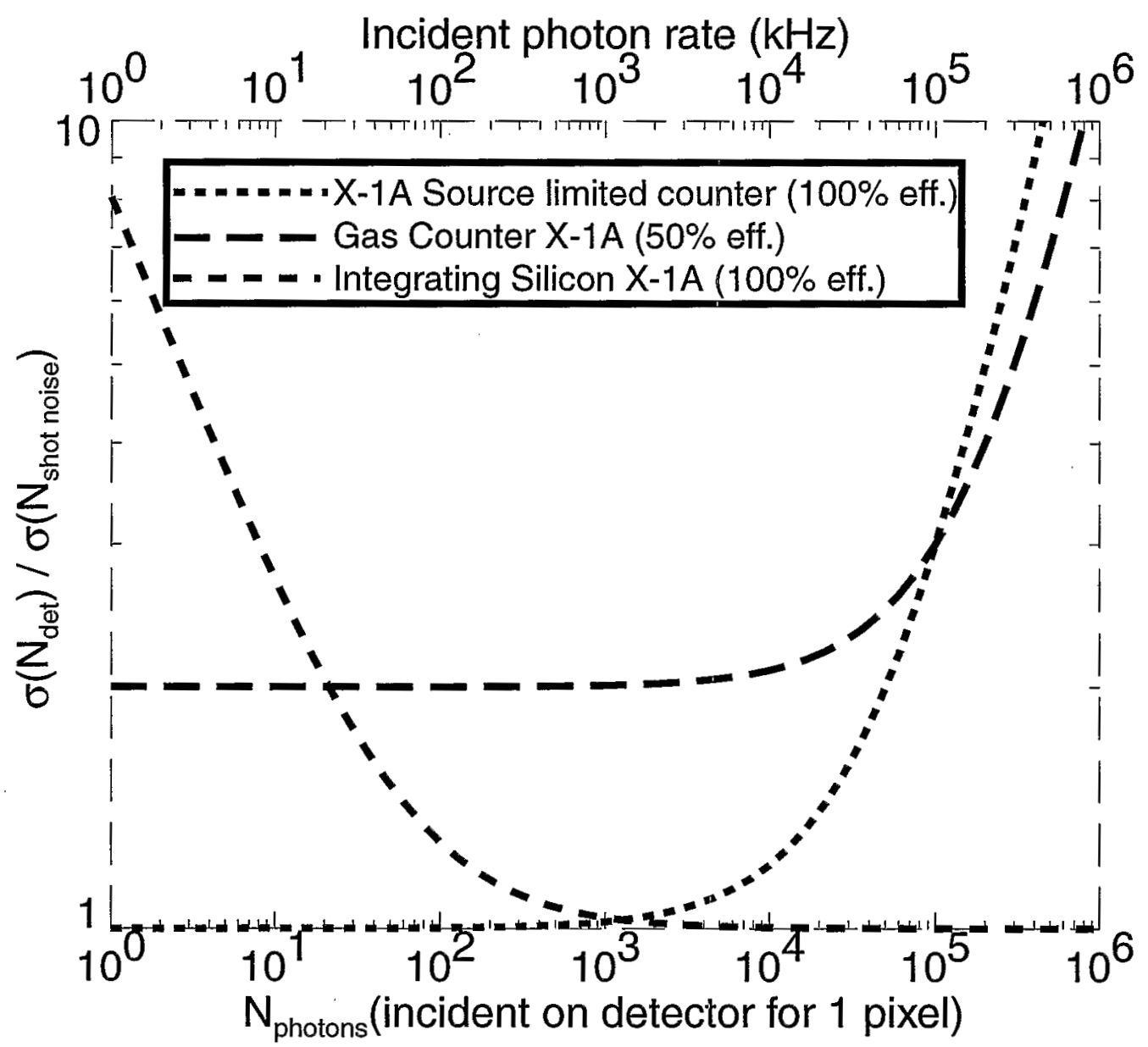

Figure 1: Comparison of counting and integrating detectors. On the vertical axis the ratio of the rms fluctuation in detected number of photons for a particular detector to the ideal detector is plotted. The ideal detector sees only fluctuations due to the photon shot noise.

The integrating detector performs more poorly at low photon numbers, but outperforms the counters at larger photon numbers due to the higher photon detection efficiency.

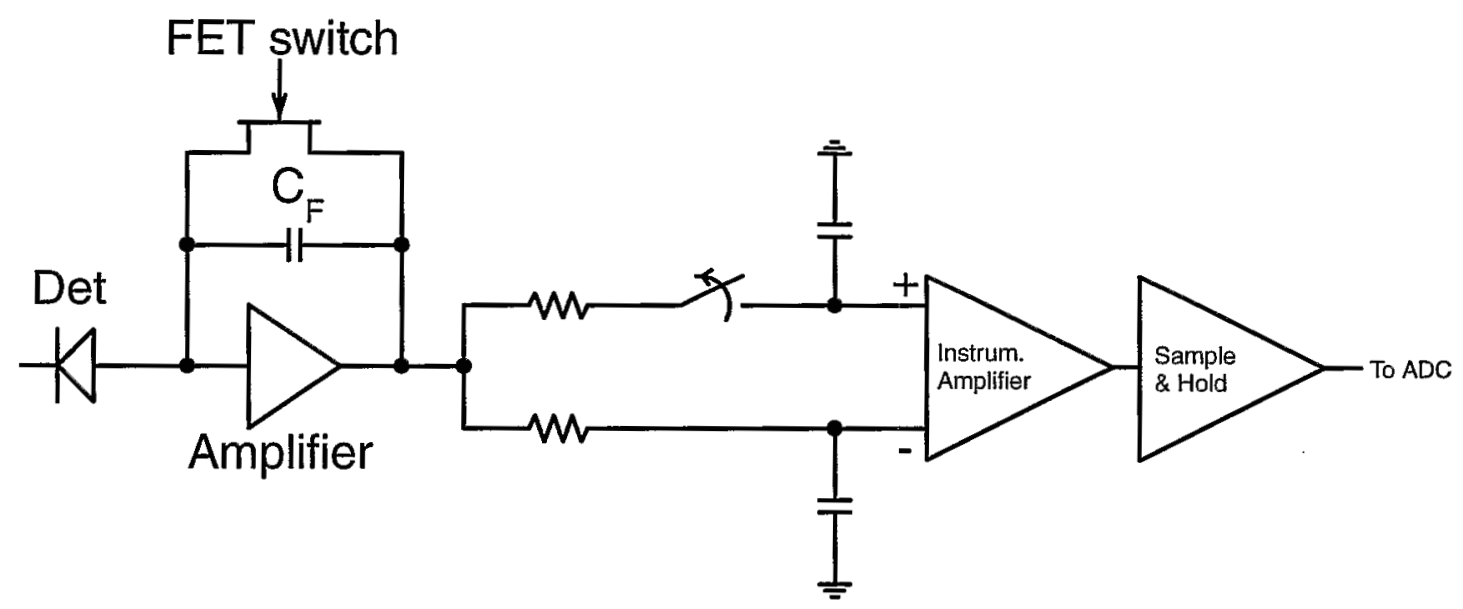

Figure 2: Simplified schematic of the integrating electronics. See text for explanation. 

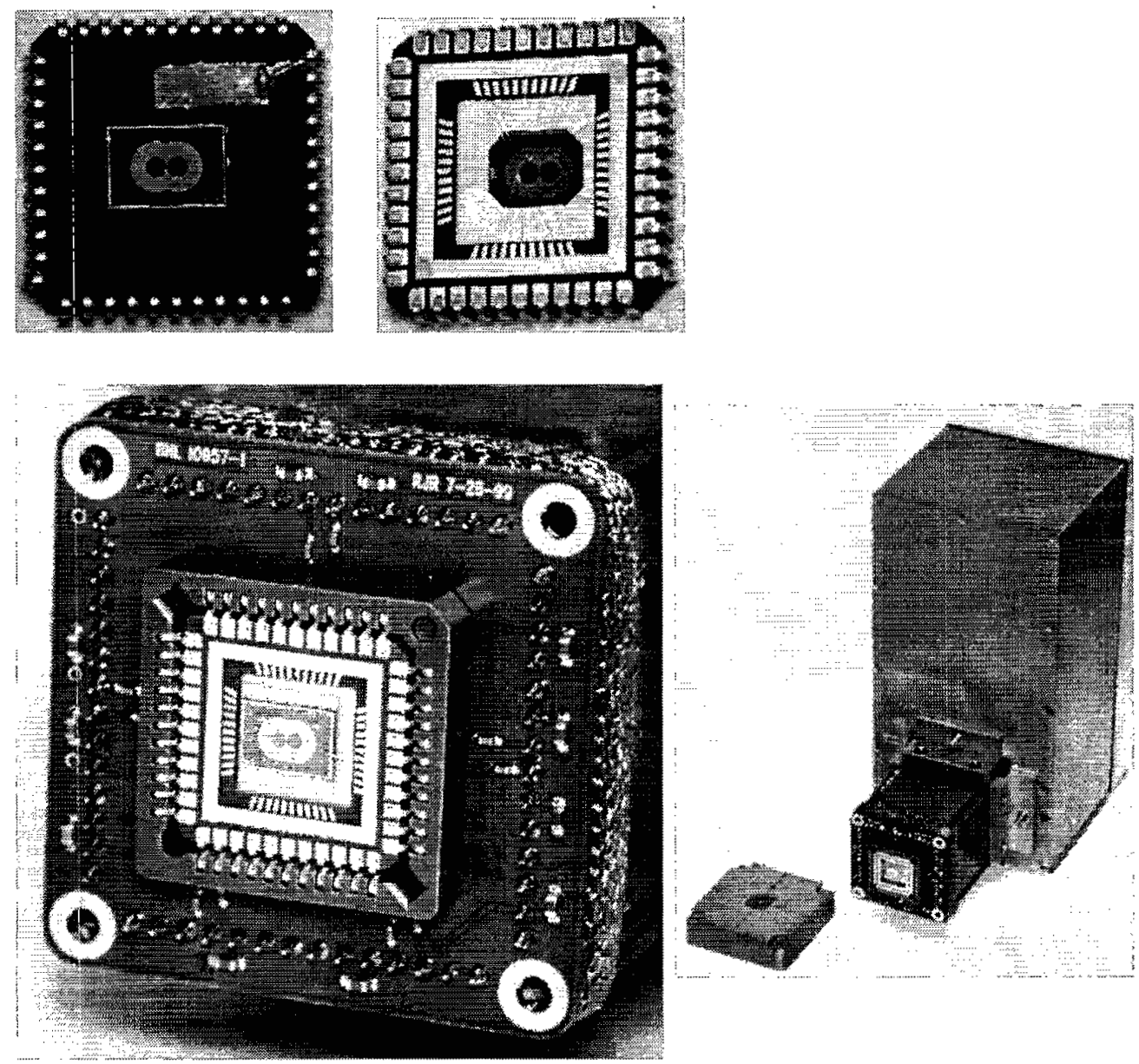

Figure 3: Images of the detector.

Upper images: Images of both sides of a ceramic carrier with a silicon chip mounted and bonded for backside illumination.

Lower images: The detector carrier mounted in the charge integrating electronics package. 


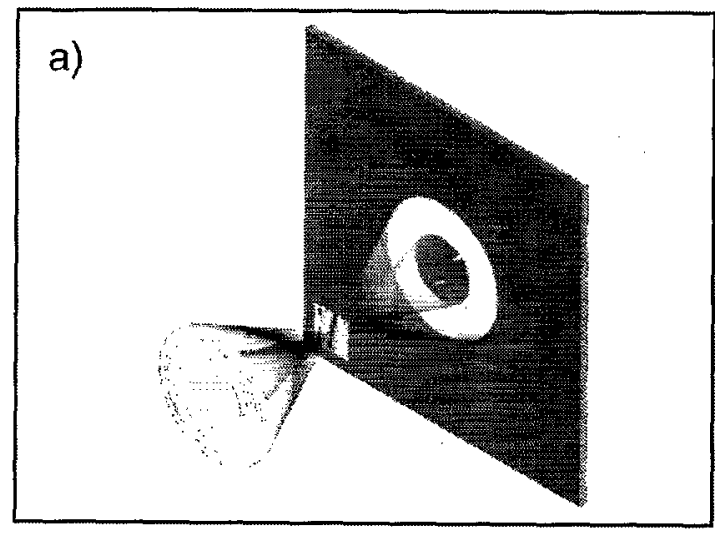

b)
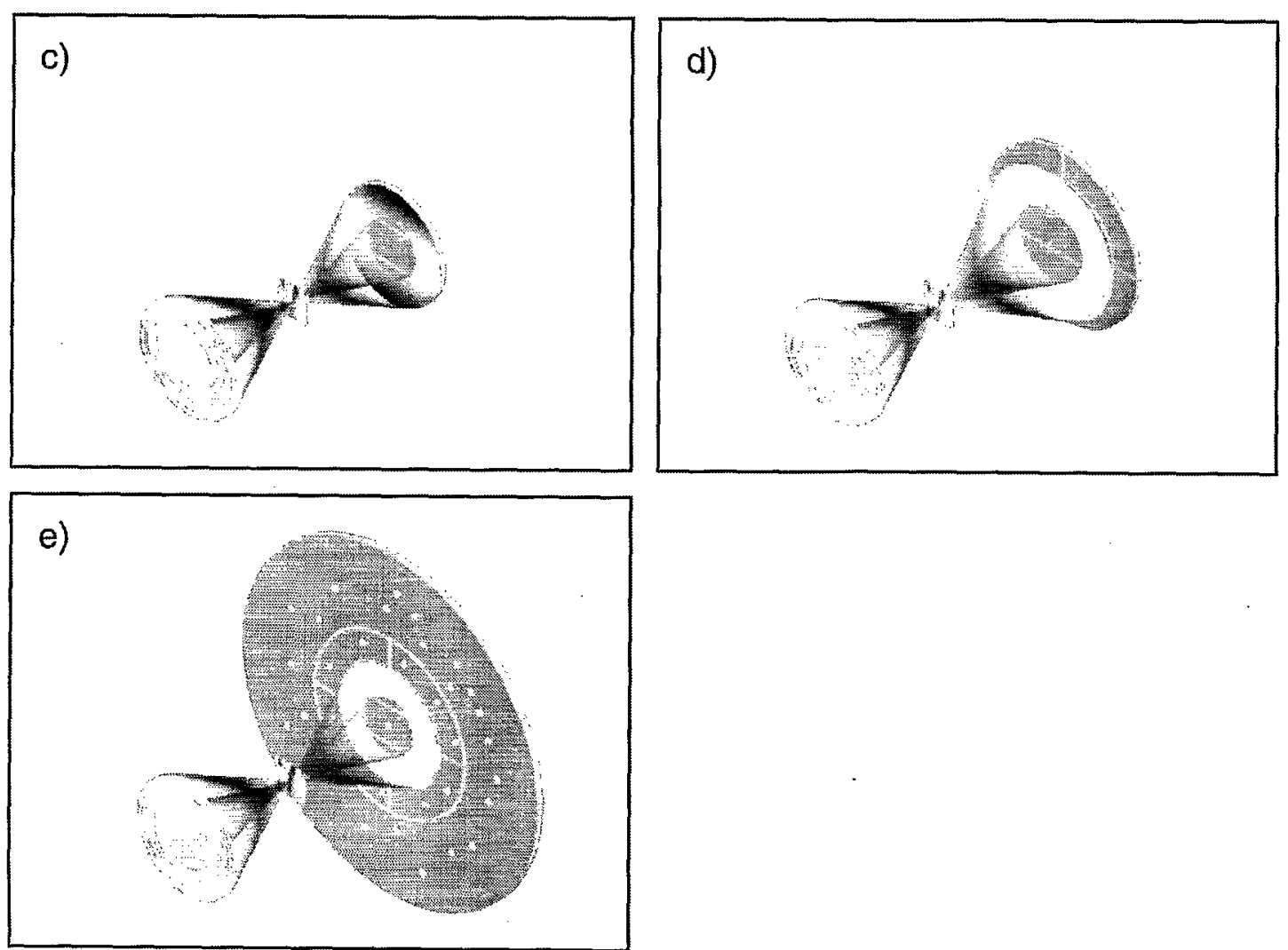

Figure 4: Illustrations of the different imaging modes in a STXM (refer to text)

a) Incoherent bright field contrast

b) Bright field with matched numerical apertures

c) Nomarski differential phase contrast (detector illumination has superimposed an interference pattern in the form of a fringe)

d) Differential interference contrast

e) Dark field contrast (white dots symbolize $\mathrm{x}$-rays scattered out of the illumination pupil) 


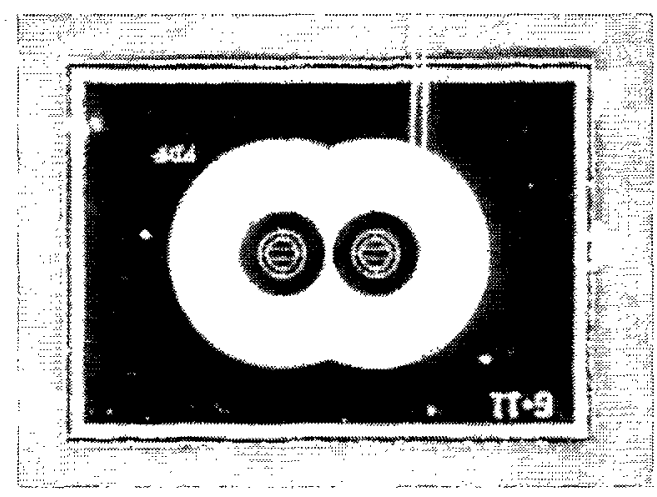

Figure 5: Image of the detector chip bonded to the ceramic carrier. This chip has been mounted for backside illumination and the pattern visible is that of an aluminum mask separating the sensitive segments. Two detector structures are visible; only one (the right one) is being used.

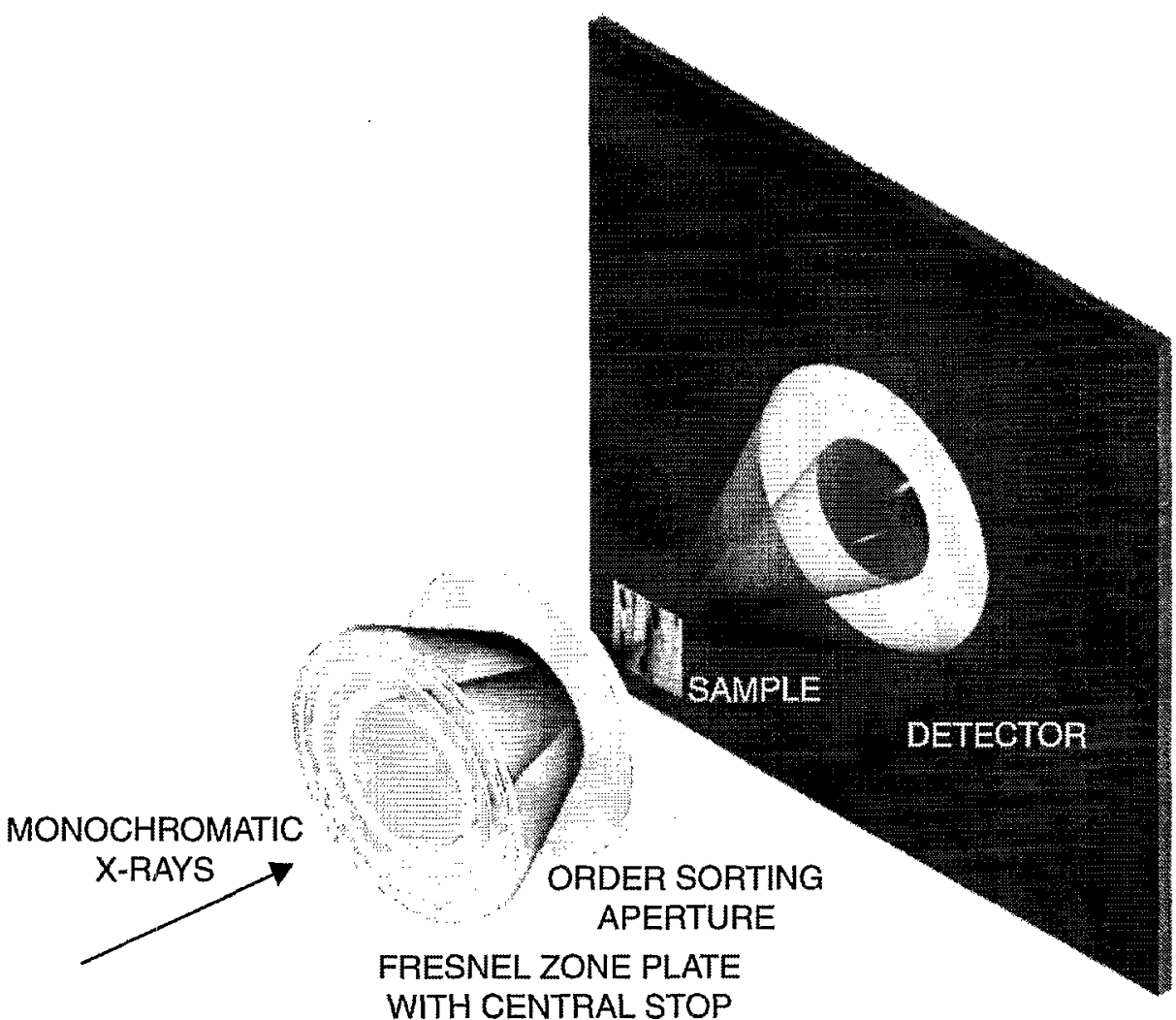

Figure 6: Illustration of the image formation in a STXM. A spatially coherent, monochromatic beam of x-rays is focused by a Fresnel zone plate into a diffraction limited spot through which the sample is raster scanned. A large central stop on the zone plate in conjunction with the order sorting aperture blocks unwanted diffraction orders from the zone plate. The transmitted x-rays are detected after the sample. 